\title{
CONE BEAM COMPUTED TOMOGRAPHIC ASSESSMENT OF MAXILLARY SINUS VOLUME VARIABILITY AMONG ADULT EGYPTIANS
}

\author{
Gihan Gamal El Din El Desouky * and Ahmad Mohamad El Rawdy**
}

\begin{abstract}
Anatomical variability in the maxillary sinus volume has been studied and reported by several researchers.
\end{abstract}

Objective: The purpose of the present study was to assess the variety in the maxillary sinus volume between genders and between the right and left sides using cone beam computed volumetric imaging.

Methods: The present retrospective study was carried on fifty cone beam computed tomography (CBCT) records of adult Egyptians aging from 20-40 years. The records were equally partitioned into two groups; the male group (G1) and the female group (G2). Volumetric examination of the antrum was conducted and statistically analyzed to compare between right and left sides and between males and females.

Results: Statistically significant differences was found in the antral volume between male and female subjects as well as between the right and left sides.

Conclusion: The volume of the maxillary sinus (MS) in male subjects was larger than the female subjects, in addition, the right-side volume was found to be larger than the left side for both genders.

KEYWORDS: Cone beam computed tomography, volumetric analysis, maxillary sinus, ondemand software.

\section{INTRODUCTION}

The maxillary sinus is considered the largest of the paranasal sinuses. It is the first of the paranasal sinuses to develop and its growth ends with the eruption of the third molars at approximately 20 years of age. The functional part of the paranasal sinuses has often been disputed. Primary hypotheses have been to add resonance to the voice; to warm and moisten air during inspiration; to play a role in mucus secretion; olfaction; and to lighten the

* Assistant Professor of Oral Radiology, Faculty of Dentistry, Suez Canal University.

** Lecturer of Oral Radiology, Faculty of Dentistry, Suez Canal University. 
skull. ${ }^{(1,2)}$ A prominent feature of the antrum is its large volume, a feature which may be related to its function. ${ }^{(3)}$

In dental implant planning, sufficient bone must be available in the upper alveolar ridge to accommodate placement of an implant and its stability. A situation which is often complicated by increase in antral size through pneumatization after exodontia of posterior teeth. Therefore, sinus augmentation procedures have been developed to provide sufficient quantity of bone for the placement of implants. To avoid unnecessary surgical complications, detailed knowledge and appropriate preoperative planning through radiographic identification of the MS anatomy and volume is required, especially when graft material is used. ${ }^{(4,5)}$

It has been suggested that the paranasal sinuses exhibit considerable left-right asymmetry in size and shape. Although the antrum is subject to fewer variations than the other air cavities, little consideration has been paid to the level of asymmetry in the maxillary sinus or to the factors associated with asymmetry. ${ }^{(6,7)}$ Therefore, the standard average size rather than correlating with the contralateral side can be used for treatment planning and assessment of the outcome in sinus surgery. ${ }^{(8,9)}$ Additionally, it is necessary to have an average index, which much corresponds to the volume. ${ }^{(10)}$

Gender determination is an essential stride in identification in forensic medicine and measurements of maxillary sinuses have been proven useful in sex determination. ${ }^{(11)}$ Although most reviews have stated that the volume of the maxillary sinuses are significantly larger in males than in females, ${ }^{(11)}$ few reported no significant sinus size differences between sexes.(12) These varying results should be grounds for further investigations.

Knowledge about the antrum volume was first carried out through taking anatomical measurements from plain radiographs. Nowadays, the introduction of three-dimensional computed tomography (CT) and magnetic resonance imaging (MRI) has allowed a more accurate assessment of this anatomical structure. ${ }^{(13)}$

The latest development in CT technology is cone beam computed tomography (CBCT) instrumentation which is a scaled-down $\mathrm{CT}$ scanner offering high resolution of bone, therefore allowing exact estimation of the maxillofacial morphology. Advantages of CBCT over traditional MDCT include considerable decrease in the degree of radiation exposure, rapid scan time, isotropic voxels offering superior spatial resolution, decreased cost, reduced image artifact and various display modes that are easily utilized by the operator and highly specific to maxillofacial imaging. ${ }^{(14-16)}$

Although computed tomography has been recommended as the most accurate method of assessment of the paranasal sinuses (17), comparisons of cone beam computed tomography (CBCT) imaging with conventional computed tomography $(\mathrm{CT})$ for pre-surgical implant planning have uncovered that $\mathrm{CBCT}$ is superior to $\mathrm{CT}$ in investigating the related anatomical structures. ${ }^{(5)}$ In addition, volumetric information acquired from a cone beam 3D survey can be accurately used to assess the sinus for visualization of anatomical borders and calculation of its actual volume. (18) Therefore, cone beam computed tomography has been chosen to be the imaging modality used the present study to calculate the volume of the maxillary sinus. The aim of this work is to evaluate the variability in the antral volume between the right and left sides as well as between males and females in a sample of Egyptian adults.

\section{PATIENTS AND METHODS}

The present retrospective study was performed on the CBCT records obtained from the archives of the outpatient clinic of the Oral Radiology Department, Faculty of Dentistry, Suez Canal University. Out of respect for patient confidentiality, all personal information concerning the patients other than gender and age was hidden. The study was carried 
out on 50 CBCT scans of bilateral maxillary sinuses (100 maxillary sinus) of both genders ranging in age from 20 to 40 years. The CBCT scans included in the present study had to reveal the whole area of the maxillary sinuses and be high quality images free from artifacts caused by metallic objects that may disable sinus visualization. Inclusion criteria also included: absence of extracted teeth in the upper posterior area, no signs of surgical procedure within the sinus, no pathological or bone lesions in the examined area and absence of mucosal enlargement in either sinuses.

The CBCT scans were equally split into two groups group I (G I); which included the scans of 25 male subjects (50 sinuses) and group II (G II); similarly included the scans of 25 female patients (50 sinuses).

\section{CBCT scanning protocol}

All CBCT scans utilized in the present study were obtained using the Scanora 3D imaging system (Sordex, Helsinki. Finland) using a CMOS flat panel detector with isotropic voxel size $133 \mu \mathrm{m}$, the $\mathrm{x}$-ray tube used to scan the patients possess a current intensity $10 \mathrm{~mA}, 90 \mathrm{Kvp}$ and a focal spot size $0.5 \mathrm{~mm}$. The scanning time was 14 seconds of pulsed exposure resulting in an effective exposure time of 3.2 seconds to scan FOV (field of view) of $14 \mathrm{~cm}$ height $\times 16.5 \mathrm{~cm}$ width. FOV adjustment was guided by three laser light beams to centralize the area of interest within the scanning field. The primary reconstruction time for DICOM data set was 2 minutes. Then, the raw DICOM data set images were imported to the On-Demand software (Cybermed, Seoul, Korea) for secondary reconstruction and image analysis.

\section{Image analysis and volumetric assessment of the maxillary sinus}

Volumetric calculation was done through "segmentation" of the maxillary sinuses, in other words, defining the region or tissue of interest. This step has to be performed before extracting any quantitative information from the $\mathrm{CBCT}$ image. In this study, semiautomatic segmentation was conducted where the software defines the boundaries of the maxillary sinus using mathematical and computer algorithms implemented within, then minimal manual adjustment was performed to remove the unwanted or include the deficient regions.

Images were examined and the region of interest (maxillary sinus) was selected to be included in the three orthogonal planes (axial, coronal and sagittal). (Fig1)

The volume of the empty space within the maxillary sinus was determined using "thresholding" level. This was done by limiting the window level to allow only the structures having CT number same like air or less to be apparent in the DICOM data set of the segmented part.

"Region growing" was used to define the maxillary sinus from the image through marking a point called a seed point in the axial cut, then the software automatically grows a 3D volumetric region of interest by comparing the adjacent volume elements (voxel) with the seed point in terms of their image intensity characteristics and including only voxels with intensity similar to that of the seed point within the region of interest. ${ }^{(19)}$ At the end of the growing process, the interior of the sinus was highlighted. (Fig 2)

Minimal manual correction (fine-tuning) was performed in the three orthogonal planes to remove the unwanted (neighboring air spaces or foramina) or include the deficient regions. ${ }^{(20)}$

Finally, the segmented highlighted volume of the maxillary sinus was then displayed as a threedimensional image, with the volume of the sinus calculated in cubic centimeters (cc) (Fig 3).

The volume of the right and left maxillary sinuses for all scans of subjects in groups I and II were measured and tabulated. 

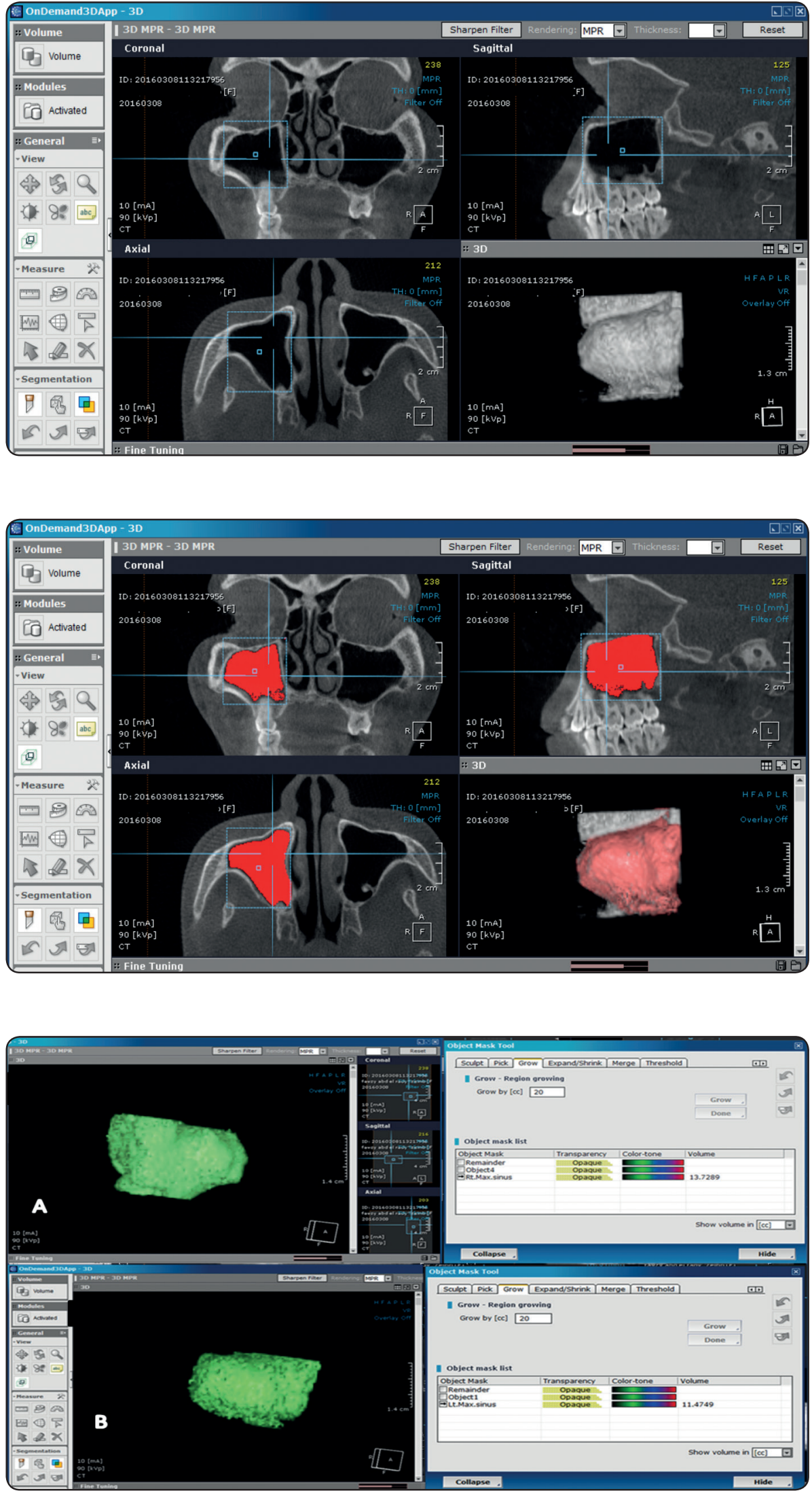

Fig (1): The software main screen revealing adjustment of the area of interest (maxillary sinus) in the axial, coronal and sagittal planes to include the whole sinus.

Fig (2): CBCT image revealing a defined and highlighted sinus in the axial, coronal and sagittal planes according to the threshold level
Fig. (3): The right and left 3D segmented maxillary sinuses revealing the volume in $\mathrm{cc}$ 
All images were analyzed separately by two independent oral radiologists. Each performed the analysis twice with two weeks' period in between the two readings to assess the intra-observer and inter-observer agreement.

\section{Statistical analysis}

Numerical information were investigated for normality by checking the data distribution and using Kolmogorov-Smirnov and Shapiro-Wilk tests. Sinus volume data showed parametric distribution.

Repeated measures ANOVA test was used to analyze among males and females as well as to compare between the right and left sides. Bonferroni's post-hoc test was used for pair-wise comparisons when ANOVA test is significant.

Inter-observer and intra-observer agreement was assessed using Cronbach's alpha reliability coefficient and Intra-Class Correlation Coefficient (ICC).

The significance level was set at $\mathrm{P} \leq 0.05$. Statistical analysis was performed with IBM $^{\circledR}$ SPSS $^{\circledR}$ Statistics Version 20 for Windows.

\section{RESULTS}

Descriptive statistics: Descriptive statistics of sinus volume measurements (cc) in males and females for both sides are presented in table 1 .

\section{Intra-observer and inter-observer agreement:}

There was very good intra- and inter-observer agreement regarding sinus volume measurements with Cronbach's alpha values ranging from 0.990 to 0.995 for intra-observer agreement and 0.976 to 0.990 for inter-observer agreement. (Table 2) Therefore, the mean of the four readings was pooled and used for further comparisons.

TABLE (1) Descriptive statistics of sinus volume measurements (cc) in males and females for both sides

\begin{tabular}{|c|c|c|c|c|c|c|c|c|}
\hline \multirow[b]{2}{*}{ Gender } & \multirow[b]{2}{*}{ Side } & \multirow[b]{2}{*}{ Mean } & \multirow[b]{2}{*}{ SD } & \multirow[b]{2}{*}{ Median } & \multirow[b]{2}{*}{ Minimum } & \multirow[b]{2}{*}{ Maximum } & \multicolumn{2}{|c|}{$95 \% \mathrm{CI}$} \\
\hline & & & & & & & Lower bound & Upper bound \\
\hline Male & Right & 19.29 & 2.39 & 19.25 & 14.33 & 23.56 & 18.25 & 20.32 \\
\hline G I & Left & 16.80 & 2.40 & 16.65 & 12.04 & 21.36 & 15.76 & 17.84 \\
\hline Female & Right & 10.58 & 2.74 & 10.37 & 4.01 & 15.36 & 9.39 & 11.76 \\
\hline G II & Left & 8.74 & 2.39 & 8.36 & 4.22 & 13.62 & 7.71 & 9.78 \\
\hline
\end{tabular}

TABLE (2) Cronbach's alpha reliability coefficient and Intra-Class Correlation Coefficient (ICC) results for intra- and inter-observer agreement regarding sinus volume measurements

\begin{tabular}{|c|l|c|c|c|c|}
\hline \multirow{2}{*}{\begin{tabular}{c}
\multirow{2}{*}{ Gender } \\
\cline { 2 - 5 } Male
\end{tabular}} & Side & Right & 0.992 & 0.985 & \multicolumn{2}{c|}{ Inter-observer } \\
\cline { 2 - 5 } G I & Left & 0.990 & 0.980 & 0.984 & 0.976 \\
\hline \multirow{2}{*}{$\begin{array}{c}\text { Female } \\
\text { G II }\end{array}$} & Right & 0.994 & 0.989 & 0.989 & 0.954 \\
\cline { 2 - 6 } & Left & 0.995 & 0.991 & 0.990 & 0.978 \\
\hline
\end{tabular}

${ }^{\circledR}$ IBM Corporation, NY, USA.

${ }^{\circledR}$ SPSS, Inc., an IBM Company. 
Comparisons: Table (3) shows the results of comparisons between the sinus volume measurements in both right and left sides for males and females (Figure 4)

\section{- Comparison between males and females:}

Males showed statistically significantly higher mean sinus volume than females in both, the right and left sides.

\section{- Comparison between right and left sides:}

The right side showed statistically significantly higher mean sinus volume than the left side in both males and females.

TABLE (3): Mean, standard deviation (SD) values and results of repeated measures ANOVA test for comparisons between sinus volume (cc)

\begin{tabular}{|l|c|c|c|c|c|}
\hline \multirow{2}{*}{ Side } & \multicolumn{2}{|c|}{ Males } & \multicolumn{2}{c|}{ Females } & $\begin{array}{c}P \text {-value } \\
\text { (Between } \\
\text { males and } \\
\text { females })\end{array}$ \\
\cline { 2 - 6 } & Mean & SD & Mean & SD & \\
\hline Right & 19.29 & 2.39 & 10.58 & 2.74 & $<0.001 *$ \\
\hline Left & 16.80 & 2.40 & 8.74 & 2.39 & $<0.001 *$ \\
\hline $\begin{array}{c}P \text {-value } \\
\text { Between right } \\
\text { and left sides) }\end{array}$ & $<0.001 *$ & \multicolumn{2}{|c|}{$<0.001 *$} & \\
\hline
\end{tabular}

*: Significant at $P \leq 0.05$

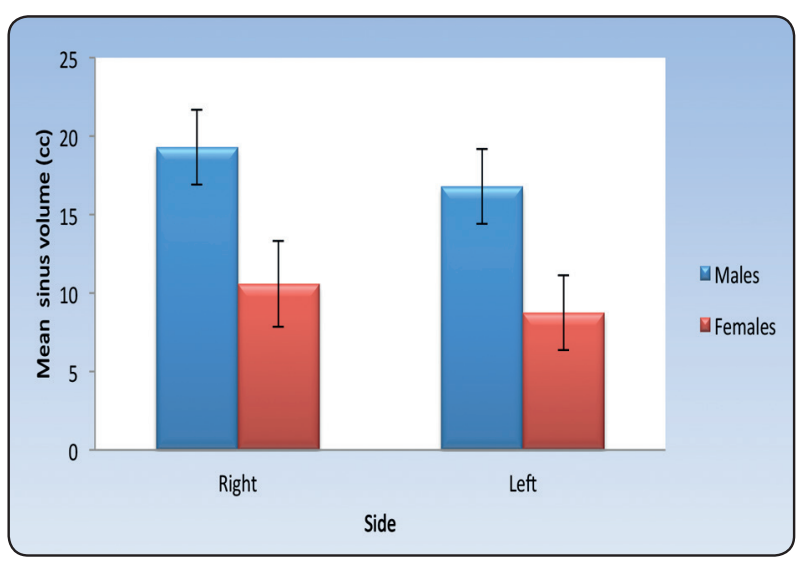

Fig. (4) Bar chart representing mean and standard deviation values of sinus volumes in both right and left sides for males and females

\section{DISCUSSION}

Accurate examination of the relation between teeth and the maxillary sinus is of extreme significance in the diagnosis and planning of endodontic surgery, orthognathic and implant procedures in the maxillary posterior region. To increase the amount of bone prior to implant placement, sinus lifting or sub-antral augmentation became a routine procedure within the last twenty years, especially when alveolar ridge atrophy or sinus pneumatization has occurred. This procedure may require placing biomaterial to reconstruct sufficient bone volume for successful implantation. Numerous variables influence the survival rates of such augmented implants, of these is the grafted bone volume. ${ }^{(21,22)}$ Preoperative knowledge of the required graft volume in addition to diagnosis of any sinus abnormalities significantly minimizes surgical time and complications. ${ }^{(23)}$

The extensive variety in maxillary sinus dimensions reported in different studies may reflect the influential effects like human, gender and race variability. ${ }^{(10,24)}$ Nevertheless, comparisons between sides and gender has often yielded conflicting results. The present retrospective study was planned to assess the volumetric measurements of the maxillary sinus in a sample of adult Egyptians of both genders to explore whether a difference existed between the right and left sides or between male and female individuals.

Only the scans of subjects over twenty years of age were incorporated in the present study to ensure that the maxillary sinus would have reached its full adult size. In addition, scans of subjects with extracted premolar or molar teeth were excluded, to prevent the expected pneumatization and change in dimensions due to invagination of the antral floor into the alveolar process following extraction, according to Havas. ${ }^{(25)}$

Cone beam computed tomography was used in our study being a reliable and accurate three dimensional technique that delivers lower radiation 
dose to the patient. ${ }^{(16)}$ Automatic segmentation with manual adjustment (semiautomatic segmentation) of images was performed for analysis of sinus volume since it provides higher accuracy than automatic segmentation, what is more, it is less subjective than manual segmentation, easier, and requires significantly less time for image analysis. ${ }^{(26-28)}$

Results of the current study revealed significant asymmetry between the right and left volume of the maxillary sinus in both male and female subjects. These results were in line with those of Anagnostopoulou et al ${ }^{(29)}$ and Nowak \& Mehlis ${ }^{(30)}$ who stated that asymmetry exists in the size of the human MS which differed mainly in whether the left or right maxillary sinus is larger. According to our results, the right maxillary sinus volume was observed to be larger than the left in both genders. In contrary, Amusa et al ${ }^{(31)}$ in 2011 studied the volume of each of the paranasal sinuses; in all the paranasal sinuses, the right side was observed to be larger than the left except for the maxillary sinus where the left side was found to be larger.

However, our results contradicted with Deeb et al, ${ }^{(19)}$ who reported no statistically significant difference in maxillary sinus volume between both sides, in addition, Hamdy and AbdelWahed ${ }^{(32)}$ assessed the bilateral linear dimensions (antroposterior and mediolateral dimensions) of the maxillary sinus and concluded that symmetric morphology was found between the two sides.

Although more studies are necessary to explore the influence of both environmental and genomic stress on the asymmetry in maxillary sinus size, Livshits and Smouse ${ }^{(33)}$ suggested that fluctuating asymmetry such as in sinus size might be considered merely as a "measure of noise" in development.

In the present study, males showed statistically significant higher mean sinus volumes than females in both the right and left sides. These results were in line with Kanthem et al ${ }^{(34)}$ who found that the dimensions and volume of maxillary sinuses were notably larger in males compared with females. They documented statistically significant values with a higher rate of sexual dimorphism in volume with $85.46 \%$ for the right side and $78.38 \%$ for the left side. Similarly, Sharma et al ${ }^{(35)}$ showed that the dimensions and volume of the maxillary sinus of males was found to be larger than those of females.

Moreover, Jasim and Al-Taei ${ }^{\left({ }^{6}\right)}$ found that the volumes and dimensions of the maxillary sinuses were larger in males than in females, in addition, the volume tends to change with advancement in age, concluding that Computed Tomography measurements of the antrum may be helpful to support gender and age determination in forensic medicine. Similarly, Jehan et al ${ }^{(37)}$ showed that the measurements of the maxillary sinuses in cone beam computed tomography (CBCT) scans can be utilized for determination of age and gender.

In addition, Vidya et al ${ }^{(38)}$ demonstrated that the measurements and volume of maxillary sinus of males were larger than females using 3D computerized tomography. Tambawala et al (39) also agreed with these results in their study using cone beam computed tomography. Most of these studies attributed the larger size of the maxillary sinus in males to sex-specific differences in body composition, weight, genetics and race. ${ }^{(40)}$

However, contradicting the results of the above studies, Ariji et al ${ }^{(12)}$ using multi detector computed tomography found that there was no significant gender difference in the volume of the maxillary sinus and there was close correlation between the right and left sides. This finding suggests the need for extra investigations in relation to the asymmetry between sides.

\section{CONCLUSIONS}

The results of the present study show that significant side and gender variation exists denoting high anatomic variability in the volume of the maxillary sinus among adult Egyptians. This finding should be always considered with care by 
maxillofacial surgeons and implantologists during sinus augmentation planning and procedures to prevent surgical complications. The semiautomatic segmentation method used in our study was found to be a time saving method for evaluation of the maxillary sinus volume.

\section{REFERENCES}

1. Misch CE. Contemporary implant dentistry. 2nd ed. St.Louis: CV Mosby Co, 1999; 76-194.

2. Rhys Evans PH. The paranasal sinuses and other enigmas: an aquatic evolutionary theory. J Laryngol Otol 1992; 106:214-25.

3. Stammberger H. Fuctional Endoscopic Sinus Surgery. Philadelphia: BC Decker. 1991; 45-7.

4. Tong DC, Rioux K, Drangshlt M, Bierne OR. A review of survival rates for implants placed in grafted maxillary sinuses using meta-analysis. Int J Oral Maxillofac Impl. 1998; 13: 175-182.

5. Nickenig HJ, Wichmann M, Zo“ller JE, Grandoch A, Eitner S, Kreppel M: Three-dimensional cone beam computed tomography analysis of maxillary sinus and alveolar bone anatomy in the restorative axis of dental implants using radiopaque drill guides. Int. J. Oral Maxillofac. Surg.2016; 45: 1485-1489.

6. Amedee RG. Sinus anatomy function head and neck surgery-otolaryngology, vol. 1. Philadelphia: Lippincott Company. 1993;. pp. 342-9.

7. Parsons PA Fluctuating asymmetry: a biological monitor of environmental and genomic stress. Heredity.1992; 68: 361-364.

8. Gunkel AR, Freysinger W, Thumfart WF. 3D anatomoradiological basis of endoscopic surgery of the paranasal sinuses. Surg Radiol Anat.1997; 19:7-10.

9. Sivasli E, Sirikci A, Bayazit YA, Gumusburun E, Erbagci $\mathrm{H}$, BayramM, et al. Anatomic variations of the paranasal sinus area in pediatric patients with chronic sinusitis. Surg. Radiol. Anat. 2002; 24:400-5.

10. Ariji Y, Ariji E, Yoshiura K, Kanda S. Computed tomographic indices for maxillary sinus size in comparison with the sinus volume. Dentomaxillofac Radiol 1996; 25:19-26.

11. Teke HY, Duran S, Canturk N, Canturk G. Determination of gender by measuring the size of the maxillary sinuses in computerized tomography scans. Surg Radiol Anat. 2007; 29 (1), 9-13.

12. Ariji Y, Kuroki T, Moriguchi S, Ariji E, Kanda S. Age changes in the volume of the human maxillary sinus: a study using computed tomography. Dentomaxillofac Radiol. Aug 1994;23 (3):163-8

13. Sanchez Fernandez JM, Anta Escuredo JA, Sanchez Del Rey A, Montoya FS. Morphometric study of the paranasal sinuses in normal and pathological conditions. Acta Otolaryngol 2000; 120:273-8.

14. Mozzo P, Procacci C, Tacconi A, et al. A new volumetric $\mathrm{CT}$ machine for dental imaging based on the conebeam technique: preliminary results. Eur Radiol.1998; 8:1558-64.

15. Hashimoto K, Kawashima S, Kameoka S, Akiyama Y, Honjoya T, Ejima K, Sawada K. Comparison of image validity between cone beam computed tomography for dental use and multidetector row helical computed tomography. Dentomaxillofac Radiol. Dec 2007; 36(8):465-71.

16. Scarfe WC and Farman AG. What is Cone Beam CT and how does it work? Dent Clin N Am. 2008; 52: 707-730.

17. Sahlstrand-Johnson P, Jannert M, Str€ombeck A, AbulKasim K. Computed tomography measurments of different dimensions of maxillary and frontal sinus. BMC Med Imaging 2011; 11(1):8.

18. Fernandes CL. Volumetric analysis of maxillary sinuses of Zulu and Europen crania by helical, multislice computed tomography. J Laryngol Otol. 2004; 118(11):877-881.

19. Deeb R, Preeti N, Malanim MD, Baljinder G, Kourosh JK, Hamid SZ and Mark AZ. Three dimensional volumetric measurments and analysis of the maxillary sinus. Am J Rhinol Allergy. 2011; 25: 152-156.

20. Gonzalez RC, and RE Woods. Morphological image processing. In Digital Image Processing. Upper Saddle River, NJ:Pearson/PrenticeHall. 2008; 627-664.

21. Hatano N, Sennerby L, Lundgern S. Maxillary sinus augmentation using sinus membrane elevation and peripheral venous blood for implant-supported rehabitation of the atropic posterior maxilla:Case series. Clin Implant Dent Relat Res. 2007; 9:150-155.

22. Tawil G and Mawla M. Sinus floor elevation using a bovine bone mineral (Bio-Oss) with or without the concomitant use of a bilayered collagen barrier (Bio-Gide): a clinical report of immediate and delayed implant placement. Int J Oral Maxillofac Implants. 2001; 16:713-721. 
23. Cordioli G, Mazzoco C, Schepers E, Brugnolo E, Majzoub Z. Maxillary sinus floor augmentation using bioactive glass granules and autogenous bone with simultanous implant, clinical and histological finding. Clinical Oral Implants Research. 2001; 12:270.

24. Koppe T, Weigel C, Ba“ renklau M, Kaduk W, Bayerlein $\mathrm{T}$, Gedrange T. Maxillary sinus pneumatization of an adult skull with an untreated bilateral cleft palate. J Craniomaxillofac Surg 2006;34 (Suppl 2):91-5.

25. Havas TE, Motbey JA, Gullane PJ. Prevalence of incidental abnormalities on computed tomographic scans of the paranasal sinuses. Arch Otolaryngol Head Neck Surg 1988;114(8):856-9.

26. Forest D, Nijjar S, Flores-Mir C, Carey J, Secanell M and Lagravere M. Comparisons of in vivo 3D cone-beam computed tomography tooth volume measurement protocols. Progress in Orthodontics.2014; 15:69.

27. El $\mathrm{H}$ and Palomo JM. Measuing the airway in 3 dimensions: A reliability and accuracy study. Am J Orthod Dentofac Orthop.2010; 137: S50-2.

28. Akkurt A, Dogru M, Hekimoglu S, Karadede I, Erdem E, Akkus M and Yilmaz Z. Three dimensional comparison of maxillary sinus volume inpatients with and without posterior crossbite. Maxillary sinus volume. 2013; 5(1):1-8.

29. Anagnostopoulou S, Venieratos D, Spyropoulos N. Classification of human maxillary sinuses according to their geometric features. Anatomischer Anzeiger; 1991: 173, 121-130.

30. Nowak R, Mehlis G. Untersuchungen zum Verhalten der Pneumatisation des Sinus maxillaris. Anatomischer Anzeiger (Investigations on the behaviour of the pneumatisation of the maxillary sinus.Anatomical indicator). 1975; 138: 143-151.

31. Amusa YB and et al. Volumetric measurements and anatomical variants of paranasal sinuses of Africans
(Nigerians) using dry crania. International journal of medicine and medical sciences. 2011; 3 (10): 299-303.

32. Reham M. Hamdy and Nagla'a Abdel-Wahed. Three-dimensional linear and volumetric analysis of maxillary sinus pneumatization. J Adv Res. 2014; 5(3): 387-395.

33. Livshits G, Smouse PE Multivariate fluctuating asymmetry in Israeli adults. Human Biology. 1993; 65: 547-578.

34. Kanthem RK, Guttikonda VR ,Yeluri S, and Kumari G. Sex determination using maxillary sinus J Forensic Dent Sci. 2015 May-Aug; 7(2): 163-167.

35. Sharma SK, Jehan M and Kumar A: measurments of maxillary sinus volume and dimensions by computed tomography scan for gender determination. Journal of Anatomical society of India. 2014; 63:36-42.

36. Jasim HH and Al-Taei JA. Computed tomographic measurement of maxillary sinusvolume and dimension in correlation to the age and gender (comparative study among individuals with dentate andedentulous maxilla). Journal of Baghdad College of dentistry. 2013; 25 : (1).

37. Jehan M, Bhadkaria V, Trivedi A, Sharma SK. Sexual dimorphism of bizygomatic distance \& maxillary sinus using CT Scan. IOSR-J Dent Med Sci. 2014;13(3):91-5.

38. Vidya CS, Shamasundar NM, Manjunatha B, Raichurkar $\mathrm{K}$. Evaluation of size and volume of maxillary sinus to determine gender by 3D computerized tomography scan method using dry skulls of South Indian origin. Int J Curr Res Rev. 2013; 5:97-100.

39. Tambawala SS, Karjodkar FR, Sansare K, and Prakash N. Sexual dimorphism of maxillary sinus using cone beam computed tomography. Egyptian Journal of Forensic Sciences. 2016; 6: 120-125.

40. Paknahad M, Shahidi S, and Zarei Z. Sexual Dimorphism of Maxillary Sinus Dimensions Using Cone-Beam Computed Tomography. J Forensic Sci. 2016. 